\title{
Tidal Volume Variability and Respiration Rate Estimation Using a Wearable Accelerometer Sensor
}

\author{
Atena Roshan Fekr, Katarzyna Radecka and Zeljko Zilic \\ Department of Electrical and Computer Engineering, McGill University, Montreal, Canada \\ atena.roshanfekr@mail.mcgill.ca, \{katarzyna.radecka, zeljko.zilic\}@ mcgill.ca
}

\begin{abstract}
The measurement of respiration rate and tidal volume variability are critical to the diagnosis and monitoring of a wide range of breath disorders as well as being useful broader parameters of a patient's condition. This paper presents a portable real-time platform designed to support a computationally efficient human respiratory tracking system for medical applications. The proposed system is designed particularly for patients with breathing problems (e.g. respiratory complications after surgery) or sleep disorders. We introduce the use of accelerometer sensor to detect changes in the anterior-posterior diameter of the chest; whereas these changes provide an accurate measurement of respiration rate as well as tidal volume variability. The complete system was comprised of wearable calibrated accelerometer sensor, Bluetooth Low Energy (BLE) and cloud database. The experiments are conducted with 8 subjects and the overall error in respiration rate calculation is obtained $0.2 \%$ considering SPR-BTA spirometer as the reference. We also present a method for Tidal Volume variability (TVvar) estimation while validated using Pearson correlation. The mean value of the correlation coefficient between TVvar derived from the accelerometer and spirometer for all subjects and three breath patterns is $\mathbf{0 . 8 7}$ which shows a high correspondence of two signals. Furthermore, the results indicate that the accelerometer driven TVvar achieves the average MSE $1.6 \mathrm{E}-03 \pm 3.69 \mathrm{E}-03$ compared to the reference.
\end{abstract}

Keywords-respiration rate; tidal volume variability; accelerometer; Bluetooth Low Energy

\section{INTRODUCTION}

The measurement of human respiratory signal is crucial in cyber biological systems. There are different respiratory disorders such as heart failure, cardiac arrhythmias, and atherosclerosis which can be the first symptom of different physiological, mechanical, or psychological dysfunctions [1]. A real-time monitoring of the respiration rhythm as well as breath patterns plays an important role in both diagnosis and treatment of different disorders. Measurements of interest may include respiratory rate, breathing pattern, and changes in tidal volume over time. All these parameters can be measured by direct methods which require breathing through some devices such as spirometer and nasal thermocouples [2]. There are also other traditional methods such as impedance plethysmography [3], pneumatic respiration transducers, the fiber-optic sensor [4], the Doppler radar [5], and electrocardiogram (ECG)-based derived respiration measurements [6]-[8]. However, these techniques are obtrusive and are not practical to be included in a body sensor network for continuous monitoring outside of hospital environments. Moreover, changes in resting respiratory rate and tidal volume are recognized as important indicators of physical health or the exacerbation of medical conditions, such as chronic obstructive pulmonary disorder (COPD). The respiratory rate is commonly assessed by visual observation in medical centers, which is not practical for remote monitoring and is also prone to error [9]. Therefore, designing a portable respiratory platform is needed for monitoring outside clinical environs. An accurate data representative of a subject's natural behavior over extended periods of time (whole day or even a week) would be of important utility in this realm. Another potential application of such a monitoring system is continuous tracking of patients after major surgery. Indeed, the respiratory complications after surgery can be severe, and in some cases, even fatal. Opioidinduced respiratory depression is a risk factor after surgery, for which irregularity of breathing and a rise in tidal volume variability are good indicators [10]. One recent area of interest is applying motion sensors to detect the small movements of the body that occur during expansion and contraction of the lungs. A validation of respiratory signal derived from suprasternal, notch acceleration has been investigated by [12] for different body positions. They show that the respiration rate from the accelerometer has $1.55 \%$ error with respect to the spirometer. Their data storage and processing is performed on a computer with their custom build LabVIEW Virtual Instrument. In [12] the respiratory component is also extracted from the accelerometer mounted on the suprasternal notch of subjects. The vibrations are recorded with a transducer electronic data sheets (TEDS) lightweight piezoelectric accelerometer. Their results represent the feasibility of implementing an accelerometry based portable device for respiration recordings. The data acquisition is done with a compact system and a laptop where data were stored to be used later. Recently, [13] introduced a fusion algorithm for accelerometer and gyroscope signals to calculate the respiration rate. They considered two types of exercises, and the respiration rate errors are calculated as $4.6 \%$ and $9.54 \%$ for the treadmill and leg press, respectively.

The main objective of this study is to provide a cloud-based tool for accurately monitoring the respiration patterns of the patients with an accelerometer sensor. The previous systems often require a laptop, memory card or handheld PC to be carried by the subject due to the processing, data storage, and power requirements of the sensory equipment. However, in our system we use cloud database which can offer significant advantages over traditional methods, including increased online accessibility, scalability, automatic failover and fast automated recovery from failures. The accelerometer data is 
transmitted via Bluetooth Low Energy (BLE) to $\mathrm{PC} /$ Smartphone and then is sent to the cloud to be processed and saved, immediately. It is worth mentioning that in case of network disconnection, the data is saved on the intermediate interface. Therefore, the physicians can track the patients wherever they are with devices such as an iPhone, iPad or the web regardless of their proximity to the patients.

Moreover, the accuracy of respiration rate compared to previous works [12], [13] has been improved in view of rest condition and moderate movements. Since, to the best of our knowledge, there is no accurate device to remotely measure the tidal volume variability and direction of the changes in $V_{T}$, in this paper we also propose an efficient technique to achieve $T V_{v a r}$ from outside of medical centers. Fig. 1 briefly described the proposed respiration monitoring platform.

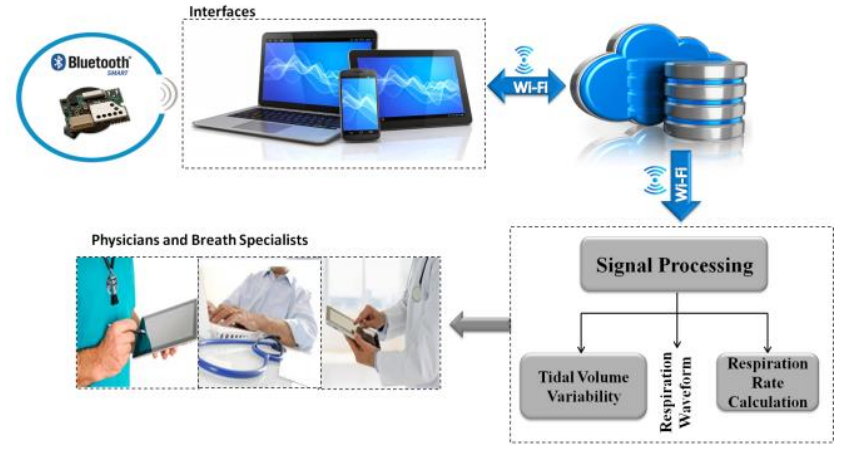

Fig. 1. Overall view of the proposed cloud-based respiration monitoring platform

In section II, a procedure is presented to accurately calculate the respiration rate. In section III, Tidal Volume Variability $\left(T V_{\text {var }}\right)$ has been investigated from the accelerometer respiration signal. Experimental results are presented and discussed in Section IV. Finally Section V concludes the paper.

\section{RESPIRATION RATE ANALYSIS}

In this section, a procedure is described to estimate the respiration rate at rest positions. In order to deliver high accuracies for the sensor measurements, we first perform a calibration technique using least square method proposed by [14]. Calibration, which is defined as the process of mapping raw sensor readings into corrected values [15], is critical in cyber-biological systems due to inherent deficiency or aging problems.

Generally, different special tools with specialists experience are required for sensors calibration; however, a straightforward method to calibrate an accelerometer is performed at 6 stationary positions [14]. We need to collect a few seconds of accelerometer raw data at each position. Then the least square method is applied to obtain the 12 accelerometer calibration parameters. The calibration procedure is simple, and needs to be executed once. The calibration procedure can be briefly explained as follows:

$$
\left[\begin{array}{lll}
a_{x} & a_{y \prime} & a_{z \prime}
\end{array}\right]=\left[\begin{array}{lll}
a_{x} & a_{y} & a_{z}
\end{array}\right]\left[\begin{array}{lll}
a c c_{11} & a c c_{21} & a c c_{31} \\
a c c_{12} & a c c_{22} & a c c_{32} \\
a c c_{13} & a c c_{23} & a c c_{33} \\
a c c_{10} & a c c_{20} & a c c_{30}
\end{array}\right]
$$

Where:

$$
\boldsymbol{y}=w \cdot X
$$

- Vector $\boldsymbol{w}$ is accelerator sensor raw data collected at 6 stationary positions

- Vector $\boldsymbol{y}$ is the known normalized Earth gravity vector.

- Matrix $\boldsymbol{X}$ is the 12 calibration parameters that is determined as below:

$$
X=\left[\boldsymbol{w}^{T} \cdot \boldsymbol{w}\right]^{-1} \cdot \boldsymbol{w}^{T} \cdot \boldsymbol{y}
$$

Our analysis is based on the acceleration signals recorded with a three-axis accelerometer, mounted on the subject's chest. Due to the location of the sensor, the major direction is $\mathrm{z}$-axis which clearly reflects the anterior-posterior motions of the chest. To remove the disruption movements, the raw sensor data is filtered through a 10th order, Butterworth (IIR) low pass filter with cut of frequency $1 \mathrm{~Hz}$. Fig. 2(a) depicts a part of respiration flow from spirometer for normal breathing pattern of a 29 years old man. We apply a numeric integration algorithm in which the trapezoidal rule was used to estimate the area under the flow curve and obtain the respiratory volume drawn in black in Fig. 2(b). As can be seen, even though two signals seem similar, there is an unwanted cumulative error [13] over time affected on their synchronization. Indeed, this type of error on signals is due to different sampling frequencies. Although, the sampling rates of accelerometer and spirometer are both set to $50 \mathrm{~Hz}$, due to architecture of the inertial measurement unit (IMU), there might be a small difference between the sampling rate and measured frequency. Thus, to estimate the respiration rate, it is essential to ensure that both signals have identical frequencies. For this purpose after rational fraction estimation, we resample our data by an anti-aliasing low pass FIR filter during the resampling process. In our experiments, the sampling rate of the accelerometer sensor was set to $50 \mathrm{~Hz}$, however; the data was logged with about $51 \mathrm{~Hz}$ (measured frequency). With resampling process explained above, we could compensate the time lead about 0.02 per second (Fig. 2).

Note that the system automatically checks the number of samples in each analysis window to find the measured frequency of the sensor. To find the best starting point between accelerometer and spirometer, the peak of their cross correlation is considered. Now, the respiration rate can be computed based on the number of local maxima in the breath signals per minute.

\section{TIDAL VOLUME VARIABILITY ESTIMATION}

The measurement of tidal volume variability requires devices such as spirometer or pneumotachometr connected to the patient with a mouthpiece and nose clip or face masks. These types of equipments are uncomfortable, and may cause a sensation of smothering [16].Therefore, a monitoring method with simple setup and good accuracy for remotely estimating $T V_{v a r}$ on a breath-by-breath basis is crucial. [17] shows that increased tidal volume variability in children is a better sign of opioid-induced respiratory depression than decreased 

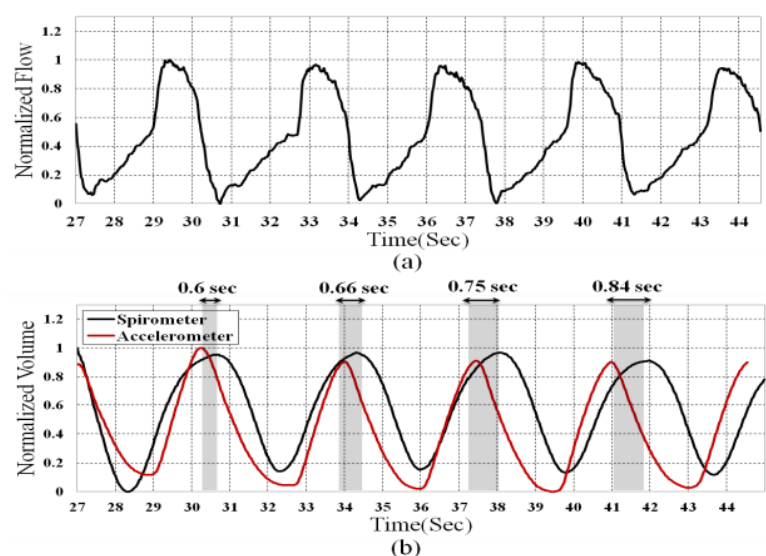

(b)

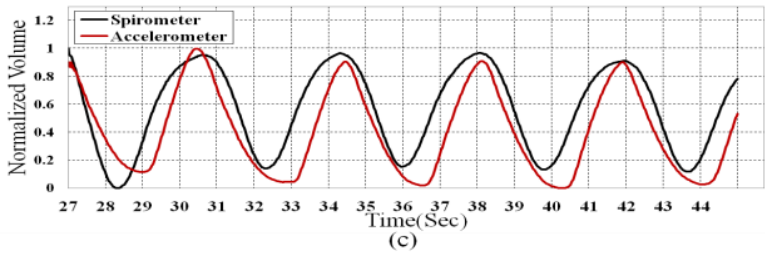

Fig. 2. (a) The normalized flow of spirometer, (b) The cumulative error of accelerometer and spirometer normalized volume signals over time before resampling (c) Two signals after resampling procedure.

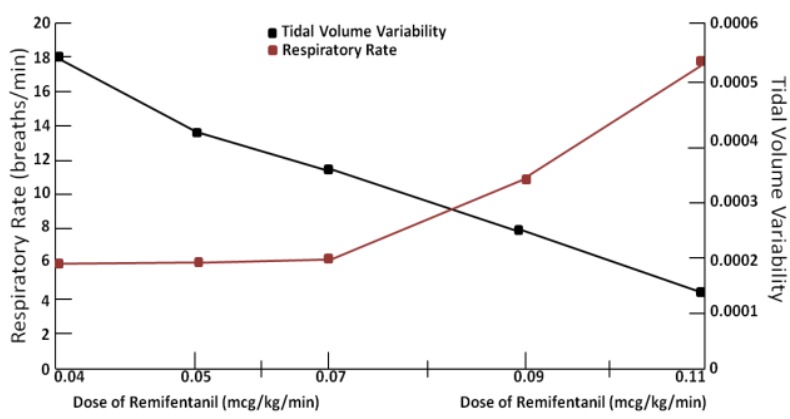

Fig. 3. Tidal volume and respiratory rate versus the dose of Remifentanil [17]

respiratory rate. It is due to the fact that an increasing in $T V_{v a r}$ is 10 times of a drop in respiratory rate. Besides, rising in $T V_{\text {var }}$ also correctly predicts respiratory depression twice as often as decreased respiratory rate. Therefore, $T V_{v a r}$ is more sensitive than the respiratory rate and the magnitude of change is larger. It is worth noting that tidal volume variability is independent of age-related variations unlike respiratory rate [17]. Fig. 3 shows the tidal volume variability and respiratory rate along with increasing doses of remifentanil in a typical patient. [18] demonstrates that an increase in tidal volume variability correctly identified that the next dose would cause respiratory depression in $41 \%$ of patients. The respiratory rate less than 10 breaths per minutes correctly identified imminent respiratory depression in only $22 \%$ of the patients [18].

Hence, monitoring of tidal volume changes provides early identification and timely treatment of exacerbations with decreasing the hospital admissions, disease costs and slow deterioration. In this paper, we introduce a new method to accurately estimate $T V_{\text {var }}$ with a single accelerometer. The accelerometer is attached on the chest to measure the inclination changes due to breathing. These changes correlated closely with tidal volume variability. The spirometer and accelerometer respiration signals are first normalized and then the volume of each breath is calculated from Eq. 1 .

$$
V_{i}=p_{i}-\frac{\left(v_{i}+v_{i+1}\right)}{2}
$$

$V_{i}$ is the volume of the $i_{t h}$ breath derived from the normalized signals, $p_{i}$ and $v_{i}$ are the $i_{t h}$ peak and valley, respectively. The calculated volumes are linearly fitted with different window sizes to obtain the trends of oscillations for both spirometer and accelerometer. The window size refers to the number of breaths to obtain the tidal volume trend. We make use of the linear least-square curve fitting of the first degree for obtaining $T V_{\text {var }}$ as follow:

$$
T V_{v a r}^{i}=\frac{t \sum_{j=1}^{t} n_{i} V_{i}-\left(\sum_{j=1}^{t} n_{i}\right)\left(\sum_{j=1}^{t} V_{i}\right)}{t \sum_{j=1}^{t} n_{i}^{2}-\left(\sum_{j=1}^{t} n_{i}\right)^{2}}
$$

Where $n$ is the breath number and $T V_{v a r}$ is obtained based on the slope of the linear fits for window size $t$. The window size should be chosen wisely based on the required sensitivity prescribed by the doctor. The proposed tidal volume estimation algorithm is outlined in Fig. 4. It shows about $45 \mathrm{sec}$ of the accelerometer and spirometer signals of Biot's respiration for a 29 years old female. Per breath tidal volumes are first obtained from the normalized signals in Fig. 4(c) and (d) while the window size is set to 3 and incremented by 2 . Then in Fig.4 (e) and (f) the least square fitted lines of each window as well as the slopes of them are shown. Finally, $T V_{v a r}$ is obtained as Fig. 4(g) from accelerometer sensor and (h) for SPRBTA spirometer. Indeed, it indicates that there are negative and positive peaks of tidal volume in the 4th and 7th windows, respectively. These changes are due to the rapid respiration epochs followed by regular periods of apnea (Fig.4 (a), (b)) in Biot's respiration pattern.

\section{EXPERIMENTAL RESULTS}

\section{A. Test Setup}

The participants of this study were 4 males and 4 females aged 18 to 46 . They were instructed how to perform each breath exercise before their recording sessions. The experimental trials lasted for about 45 minutes per subject. We asked the subjects to perform Normal, Bradapnea, Tachypnea, and Kussmaul patterns, each for 1 minute, Cheyn-stokes and Biot's each for 2 minutes and finally a pattern with different tidal volume lasted for about 3 minutes. We assigned a 3minute rest interval after performing each pattern. Based on the definitions, for Normal breathing we consider 12 to $20 \mathrm{rpm}$ (respiration per minute), in Bradapnea less than $12 \mathrm{rpm}$ and for Tachypnea the subjects are asked to breathe more than 20 respirations per minutes. For simulating apnea in Cheynstokes and Biot's breathing exercises, we requested the participants to pause breathing for at least 20 seconds.

In our system we used an ultra low-power LIS3DH 3-axis accelerometer with 12-bit resolutions. In our tests the sampling rate is set to $50 \mathrm{HZ}$. 


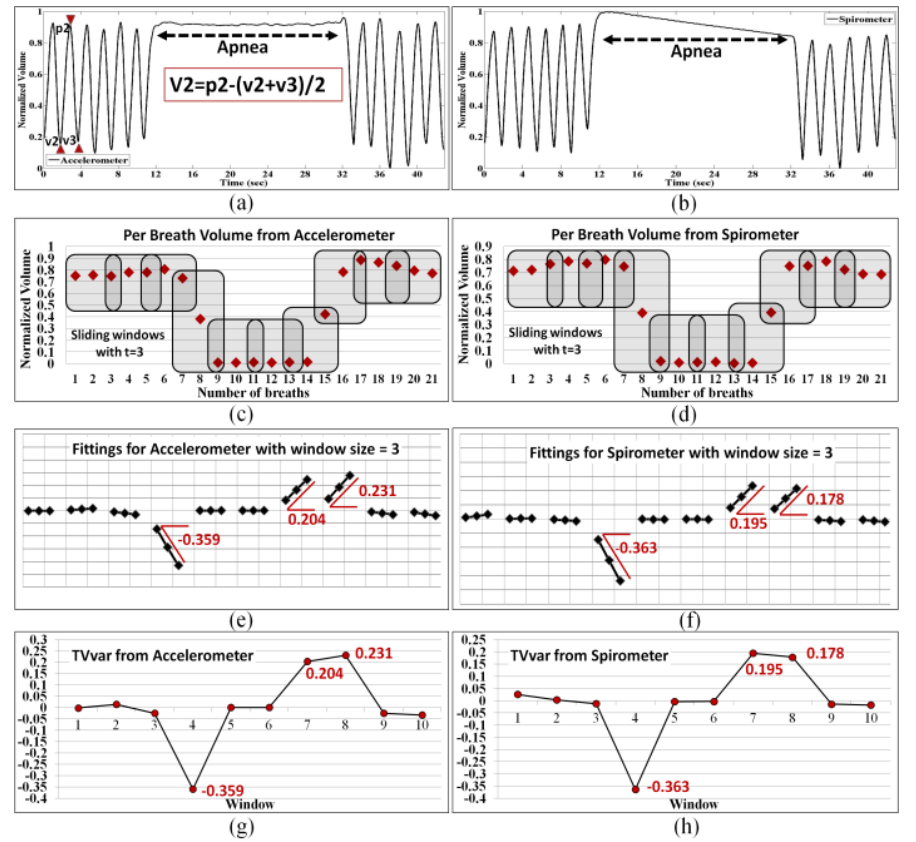

Fig. 4. (a), (b) The normalized volume from accelerometer sensor and spirometer of Biot's pattern, (c), (d) Per breath tidal volume after accelerometer signal and spirometer normalization, (e), (f) The linear fittings of accelerometer and spirometer driven per breath tidal volume with $\mathrm{t}=3,(\mathrm{~g})$, (h) the tidal volume variability from accelerometer and spirometer signals respectively

The sensor was mounted on the subject's chest in the middle of sternum region and secured by a soft and elastic strap which is easy to attach and comfortable to wear. In the trial session, the subjects were in the lying position; however, the rest positions or activities in which rib cage is stationary could be considered.

\section{B. Accelerometer Driven Respiration Rate Validation}

In this section, first the correlation between the spirometer and accelerometer signal is calculated on 8 different subjects with various ages, each for five types of breathing disorders. Next, the respiratory rate which recognized as important indicators of physical health or the exacerbation of medical conditions is calculated. In our test, the subjects are asked to perform six breathing patterns i.e. Normal, Bradapnea, Tachypnea, Cheyn-stokes, Kaussmal, and Biot's. Bradapnea is regular in rhythm but slower than normal in rate. Tachypnea is the condition of rapid breathing, with respiration rate higher than $20 \mathrm{rpm}$. Tachypnea may occur due to physiological or pathological problems [19]. Cheyn-stokes breathing pattern is determined by gradually increasing, then decreasing the lung volume with a period of apnea. People suffering from central sleep apnea syndrome (CSAS) have the same breathing pattern at sleep [20].

Kussmaul breathing which is defined as a rapid, deep and labored breathing type usually occurs in diabetics in diabetic ketoacidosis [21]. The Biot's breathing is characterized by periods of rapid respirations followed by regular periods of apnea. There are different reasons which causes Biot's breathing, such as damage to the medulla oblongata by stroke (CVA) or trauma, or pressure on the medulla due to uncal or tenorial herniation and prolonged opioid abuse [21][22]. The average correlation of spirometer and accelerometer signals of all subjects for each breath pattern is shown in Fig. 5. The correlation between accelerometer sensor (A) and spirometer (S) with $x$ samples is calculated as follows:

$$
R_{A, S}=\frac{x\left(\sum_{i=1}^{x} A_{i} S_{i}\right)-\left(\sum_{i=1}^{x} A_{i}\right)\left(\sum_{i=1}^{x} S_{i}\right)}{\sqrt{\left[x \sum_{i=1}^{x} A_{i}^{2}-\left(\sum_{i=1}^{x} A_{i}\right)^{2}\right]\left[x \sum_{i=1}^{x} S_{i}^{2}-\left(\sum_{i=1}^{x} S_{i}\right)^{2}\right]}}
$$

For instance, in Normal pattern we have 8-minute data for 8 subjects and the correlation of the accelerometer and spirometer signals is achieved 0.91. According to Fig. 5, the mean of the obtained correlations of all breathing patterns is 0.85 which shows a very close correspondence of the sensor and spirometer data. The average respiration rate errors on all subjects for each breathing patterns has been also calculated by using a 10-seconds non-overlapping sliding window. We observed that, the errors are always below $0.45 \%$ for all patterns while the overall error is obtained $0.2 \%$ compared to SPR-BTA spirometer as the reference. Thus, we could obtain better accuracy for respiration rate compared to [12], dual strain gauge respirometers [23], and [13] in lying position using a single accelerometer. The details of the experiments are brought in Table I.

\section{Tidal Volume Variability Estimation}

We consider three types of patterns for tidal volume variability evaluation. In Biot's breathing the patients breathe rapidly with high volume continuing with more than 20 seconds apnea.

TABLE I. RESPIRATION RATE MEASUREMENTS WITH BOTH ACCELEROMETER AND SPIROMETER FOR DIFFERENT SUBJECTS

\begin{tabular}{|c|c|c|c|c|c|c|c|c|c|c|c|c|c|}
\hline \multirow{2}{*}{$\begin{array}{c}\text { Subject } \\
\text { ID }\end{array}$} & \multirow{2}{*}{$\begin{array}{c}\text { Gender/ } \\
\text { Age }\end{array}$} & \multicolumn{6}{|c|}{ Respiration Rate with Accelerometer (rpm) } & \multicolumn{6}{|c|}{ Respiration Rate with Spirometer (rpm) } \\
\hline & & Normal & Bradapnea & Tachypnea & $\begin{array}{l}\text { Cheyn- } \\
\text { stokes }\end{array}$ & Kussmaul & Biot's & Normal & Bradapnea & Tachypnea & $\begin{array}{l}\text { Cheyn- } \\
\text { stokes }\end{array}$ & Kussmaul & $\overline{\text { Biot's }}$ \\
\hline 1 & F/46 & 15.22 & 6.85 & 65.86 & 21.65 & 36.11 & 27.55 & 15.30 & 6.90 & 65.86 & 21.66 & 35.91 & 27.58 \\
\hline 2 & $\mathbf{F} / \mathbf{3 0}$ & 17.41 & 9.80 & 22.14 & 24.75 & 50.23 & 32.40 & 17.41 & 9.95 & 22.26 & 24.75 & 50.23 & 32.36 \\
\hline 3 & F/29 & 13.94 & 7.46 & 22.70 & 20.36 & 39.10 & 36.38 & 13.92 & 7.39 & 22.68 & 20.36 & 39.11 & 36.36 \\
\hline 4 & M/29 & 15.55 & 7.84 & 23.26 & 14.08 & 27.49 & 36.91 & 15.56 & 7.80 & 23.33 & 14.09 & 27.54 & 36.92 \\
\hline 5 & M/28 & 14.46 & 7.42 & 35.63 & 19.94 & 40.79 & 35.42 & 14.46 & 7.64 & 35.55 & 19.95 & 40.82 & 35.46 \\
\hline 6 & $F / 22$ & 12.66 & 10.37 & 46.07 & 25.44 & 47.90 & 37.12 & 12.68 & 10.49 & 46.18 & 25.40 & 47.93 & 37.08 \\
\hline 7 & $M / 24$ & 14.44 & 10.78 & 54.19 & 22.22 & 45.25 & 37.25 & 14.42 & 10.83 & 54.16 & 22.25 & 45.34 & 37.24 \\
\hline 8 & M/18 & 13.41 & 8.19 & 50.42 & 14.58 & 45.64 & 38.41 & 13.44 & 8.13 & 50.25 & 14.63 & 45.91 & 38.44 \\
\hline
\end{tabular}




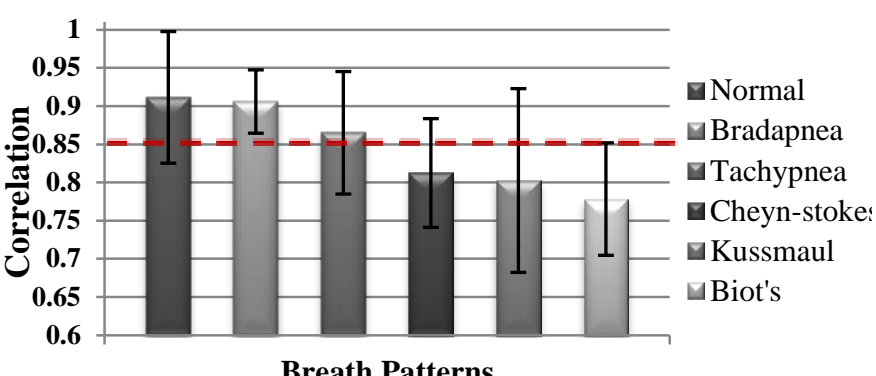

Fig. 5. Average correlation between spirometer and accelerometer signals and the standard deviations for five different patterns

We expect to have reduction in tidal volume in each apnea with no significant changes during breathing cycles. However, in Cheyn-stokes pattern the tidal volume increases gradually and then decreases to start an apnea epoch. Additionally, we analyzed the changes in tidal volume for normal to deep, deep to shallow and shallow to normal conditions (P1). Fig. 6 shows three respiration patterns for one of the subjects with his tidal volume variability obtained from accelerometer signal. There are positive changes from normal to deep as well as from shallow to normal breathings. As is shown in Fig 6(b) the variation from deep respiration to normal is less than changing pattern from deep to shallow breathings. We also obtain negative changes from deep to normal and deep to shallow breathings. The directions of the changes in $T V_{v a r}$ are always the same as the spirometer in major changes. However, the different directions are resulted from the small acceleration movement of the body during constant tidal volume. To show the impact of size, we have swept $t$ from 2 to 10 breaths. Indeed, $t$ shows the sensitivity of the system. As can be seen, there is a tradeoff between $t$ and correlation. Larger window size resulted in better correlation of accelerometer versus the spirometer. This is due to unwanted motions of the rib cage during constant tidal volume epochs. Applying larger window size removes the trends of constant tidal volume while keeping
TABLE II. AVERAGE CORRELATION BETWEEN TIDAL VOLUME VARIABILITY FROM ACCELEROMETER AND SPIROMETER OF THREE DIFFERENT PATTERNS

\begin{tabular}{|cccccc|}
\hline $\begin{array}{c}\text { Subject } \\
\text { ID/Window } \\
\text { size }\end{array}$ & $\mathbf{t}=\mathbf{2}$ & $\mathbf{t}=\mathbf{3}$ & $\mathbf{t}=\mathbf{5}$ & $\mathbf{t}=\mathbf{1 0}$ & Average \\
\hline $\mathbf{1}$ & 0.83 & 0.88 & 0.91 & 0.92 & $\mathbf{0 . 8 9}$ \\
$\mathbf{2}$ & 0.68 & 0.76 & 0.81 & 0.89 & $\mathbf{0 . 7 9}$ \\
$\mathbf{3}$ & 0.67 & 0.79 & 0.84 & 0.92 & $\mathbf{0 . 8 1}$ \\
$\mathbf{4}$ & 0.83 & 0.92 & 0.93 & 0.96 & $\mathbf{0 . 9 1}$ \\
$\mathbf{5}$ & 0.75 & 0.83 & 0.93 & 0.96 & $\mathbf{0 . 8 7}$ \\
$\mathbf{6}$ & 0.74 & 0.85 & 0.91 & 0.93 & $\mathbf{0 . 8 6}$ \\
$\mathbf{7}$ & 0.76 & 0.82 & 0.87 & 0.91 & $\mathbf{0 . 8 4}$ \\
$\mathbf{8}$ & 0.96 & 0.97 & 0.98 & 0.99 & $\mathbf{0 . 9 8}$ \\
Average & $\mathbf{0 . 7 8}$ & $\mathbf{0 . 8 5}$ & $\mathbf{0 . 9 0}$ & $\mathbf{0 . 9 4}$ & $\mathbf{0 . 8 7}$ \\
\hline
\end{tabular}

the major changes. It is worth noting that; based on our patterns introduced earlier we increase the size of window up to 10 breaths, because there are always more than 10 breaths per cycle in all of our patterns (Biot's, Cheyn-stokes and P1). Based on the respiration disorder, if the tidal volume of the patient changes frequently, the small window size is preferable; otherwise larger size accurately show the trend of tidal volume over time. Therefore, in our portable platform we could manage the appropriate sensitivity value based on different breath disorders characteristics. The correlation of $T V_{\text {var }}$ for different sizes between accelerometer and spirometer for 8 subjects are provided in Table II.

The overall correlation is obtained 0.87 whereas it proves that $T V_{v a r}$ derived from the accelerometer is strongly correlated with spirometer. The average Mean Square Errors (MSE) of tidal volume variability versus the reference for three types of patterns are 3.06E-05, 1.86E-03, 1.07E-03 and 4.33E-04 with standard deviations 7.27E-03, 4.36E-03, 2.31E03 and $8.41 \mathrm{E}-04$ for $\mathrm{t}=2,3,5$ and 10 , respectively. The errors do decrease at the larger window sizes due to less sensitivity in the constant tidal volume variability periods.

The results have shown that the MSE of each subject can
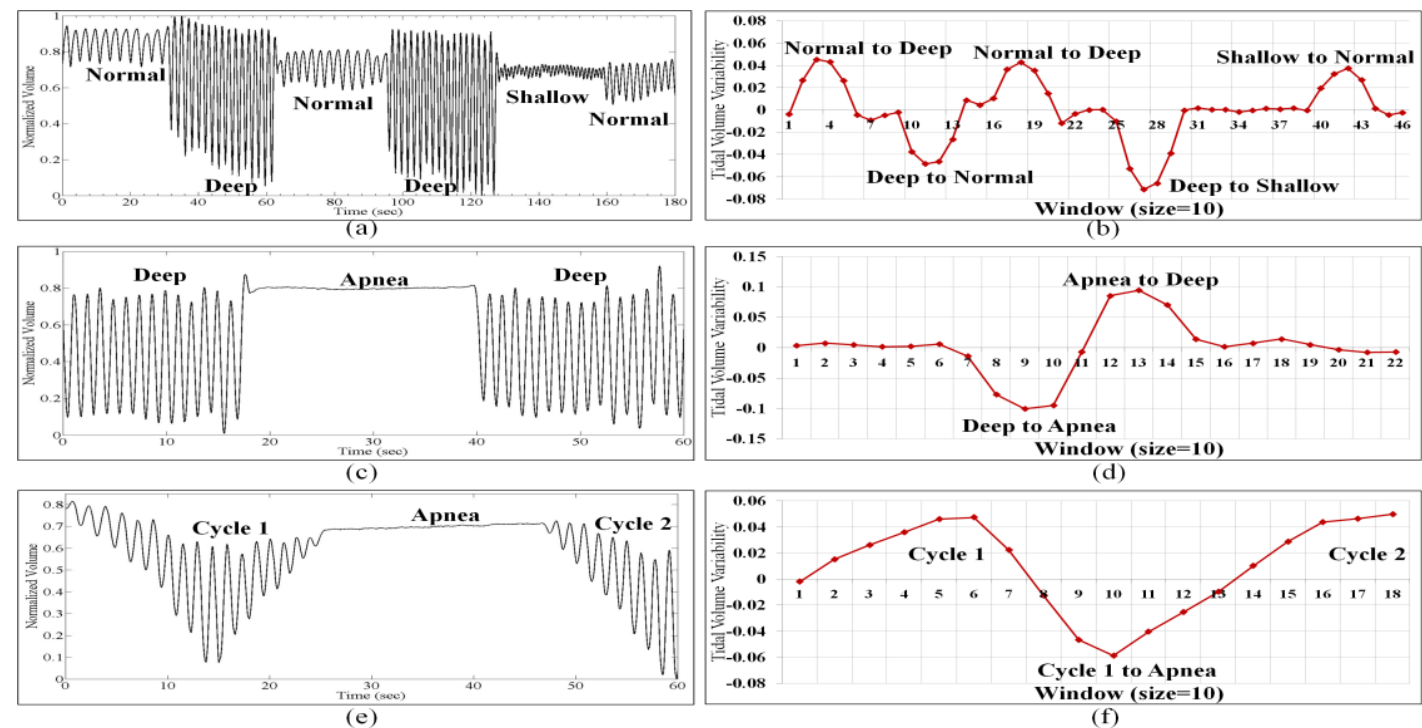

(f)

Fig. 6. (a) Pattern with different tidal volume (P1), (b)Tidal Volume Variability for P1, (c) Biot's breathing pattern, (d) Tidal Volume Variability for Biot's pattern, (e) Cheyn-stokes breathing pattern, (f) Tidal Volume Variability for Cheyn-stokes breathing pattern 
be fitted into a normal distribution, depicted in Fig. 7. In this figure the fitted normal distributions correspond to the average log-likelihood value of $124.8 \pm 28.60$ on different $t$, which indicates a good fit. Fig. 7 also shows the impact of the window size on MSE distributions. When the window is small the standard deviation is larger compared to the case with larger window sizes. Therefore, e.g. with $t=10$, the MSEs have less dispersion from the average value of zero.

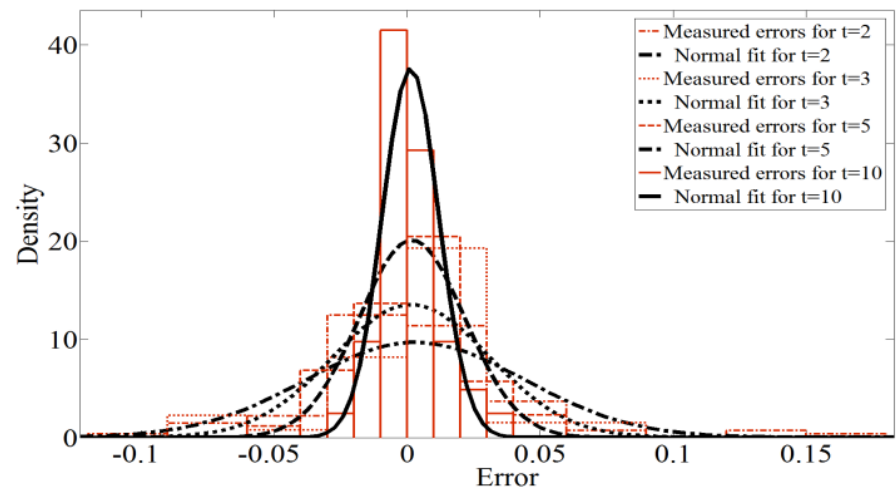

Fig. 7. Normal fittings of the mean square errors between Acceleroemter and Spirometer for Biot's breathing of S2 considering different window sizes

As can be seen, a very high correlation between accelerometer driven $T V_{v a r}$ and spirometer in all conditions coupled with significantly low MSE indicates that the proposed method is promising to be used in a respiration tracking systems specifically for real-time monitoring of breath diseases during sleep.

\section{CONCLUSION}

In this paper we have demonstrated for the first time that obtaining tidal volume variability from an accelerometer data offers a reliable and unobtrusive technique over extended periods. Furthermore, we introduced refinements to respiratory rate calculation which resulted in a very low average error of $0.2 \%$. Therefore, the possibility of acquiring both respiration rate and $T V_{v a r}$ information from one device gives more credits to accelerometer based approaches as a simple and cost effective solution for remote monitoring applications. Our focus in further will be validating the accuracy of the method especially in different moderate activities. We will also work on breath therapy concept to be added in our portable respiration platform.

\section{REFERENCES}

[1] X. Zhu, W. Chen, T. Nemoto, Y. Kanemitsu, K. Kitamura, K. Yamakoshi, and D. Wei, "Real-time monitoring of respiration rhythm and pulse rate during sleep," Biomedical Engineering, IEEE Transactions on, vol. 53, pp. 2553-2563, 2006.

[2] A. Bates, M.J. Ling, J. Mann, D.K. Arvind, "Respiratory Rate and Flow Waveform Estimation from Tri-Axial Accelerometer Data," Proceedings of the 2010 International Conference on Body Sensor Network (BSN), Biopolis, Singapore, pp. 144-150, 2010.

[3] M. R. Neuman, H. Watson, R. S. Mendenhall, J. T. Zoldak, J. M. Di Fiore, M. Peucker, T.M. Baird, D. H. Crowell, T. T. Hoppenbrouwers, D. Hufford, C. E. Hunt, M. J. Corwin, L. R. Tinsley, D. E. WeeseMayer, and M. A. Sackner, "Cardiopulmonary monitoring at home: The CHIME monitor," Physiol. Meas., vol. 22, no. 2, pp. 267-286, May 2001.
[4] L. G. Lindberg, H. Ugnell, and P. A. Oberg, "Monitoring of respiratory and heart rates using a fibre-optic sensor," Med. Biol. Eng. Comput., vol. 30, no. 5, pp. 533-537, Sep. 1992.

[5] G. Matthews, B. Sudduth, and M. Burrow, "A noncontact vital signs monitor," Crit. Rev. Biomed. Eng., vol. 28, no. 1-2, pp. 173-178, 2000.

[6] G. B. Moody, R. G. Mark, A. Zoccola, and S. Mantero, "Derivation of respiratory signals from multi-lead ECGs," in Proc. Computers in Cardiology, vol. 12, pp. 113-116, 1985.

[7] P. de Chazal, C. Heneghan, E. Sheridan, R. Reilly, P. Nolan, and M. O'Malley, "Automated processing of the single-lead electrocardiogram for the detection of obstructive sleep apnoea," IEEE Trans. Biomed. Eng., vol. 50, no. 6, pp. 686-696, Jun. 2003.

[8] D. Dobrev and I. Daskalov, "Two-electrode telemetric instrument for infant heart rate and apnea monitoring," Med. Eng. Phys., vol. 20, no. 10, pp.729-734, Dec. 1998.

[9] J. Mann, R. Rabinovich, A. Bates, S. Giavedoni, W. MacNee, D. K. Arvind, "Simultaneous Activity and Respiratory Monitoring Using an Accelerometer," Body Sensor Networks (BSN), International Conference on , pp. 139-143, May 2011.

[10] T. Bouillon, J. Bruhn, H. Roepcke, and A. Hoeft, "Opioid-induced respiratory depression is associated with increased tidal volume variability," Eur. J. Anaesthesiol., vol. 20, no. 2, pp. 127-33, Feb 2003.

[11] D.S. Morillo, J.L.R. Ojeda, L.F.C. Foix, A.L. Jimenez, "An Accelerometer-Based Device for Sleep Apnea Screening," IEEE Trans. Inf. Technol. Biomed., vol. 14, pp. 491-499, 2010.

[12] P.K. Dehkordi, M. Marzencki, K. Tavakolian, M. Kaminska, B. Kaminska, "Validation of respiratory signal derived from suprasternal notch acceleration for sleep apnea detection," Engineering in Medicine and Biology Society, EMBC, International Conference of the IEEE, pp. 3824-3827, 2011.

[13] Ja-Woong Yoon, Yeon-Sik Noh, Yi-Suk Kwon, Won-Ki Kim, Yoon Hyung-Ro, " Improvement of Dynamic Respiration Monitoring Through Sensor Fusion of Accelerometer and Gyro-sensor,"Journal of electrical engineering \& technology vol. 9, no. 1; pp. 334-343, 2014.

[14] "Tilt measurement using a low-g 3-axis accelerometer," Application note, STMicroelectronics group of companies, 2010.

[15] E. Waltz, "Data fusion for C3I: A tutorial," in Command, Control, Communications Intelligence (C3I) Handbook. Palo Alto, CA: EW Communications, pp. 217-226, 1986.

[16] R. Gilbert, J. H. Jr. Auchincloss, J. Brodsky, and W. Boden, "Changes in tidal volume, frequency, and ventilation induced by their measurement," J. Appl. Physiol. Vol.33, pp. 252-254, 1972.

[17] SJ Barbour, CA Vandebeek, JM Ansermino. "Increased tidal volume variability in children is a better marker of opioid-induced respiratory depression than decreased respiratory rate,". J Clin Monit Comput., 18:171-8, 2004

[18] J. Mark Ansermino, Kirk Shelley,"When Monitoring Analgesia in Pediatric Patients Tidal Volume Variability Can Predict Respiratory Depression," annual meeting of the Society for Technology in Anesthesia and interviews, vol. 30:09, 2004

[19] H. William, L. Myron, D. Robin, A. Mark, "Current Diagnosis and Treatment in Pediatrics," 21 Ed., McGraw-Hill Professional: New York City, USA, p. 989.

[20] K. Parveen, C. Michael, "13". Clinical Medicine, 6th Ed; Elsevier: Philadelphia, USA, p. 733

[21] R.J. Farney, J.M. Walker, K.M. Boyle, T.V. Cloward, K.C. Shilling, "Adaptive servoventilation (ASV) in patients with sleep disordered breathing associated with chronic opioid medications for non-malignant pain," J. Clin. Sleep Med., vol. 4, pp. 311-319, 2008.

[22] A. Roshan Fekr, M. Janidarmian, K. Radecka, Z. Zilic, "A medica cloud-based platform for respiration rate measurement and hierarchical classification of breath disorders," Sensors 2014, 14, 11204-11224.

[23] C. A. Kushida, M. R. Littner, T. Morgenthaler, C. A. Alessi, D. Bailey, J. Coleman, L. Friedman, M. Hirshkowitz, S. Kapen, M. Kramer, T. Lee-Chiong, D. L. Loube, J. Owens, J. P. Pancer, and M. Wise, "Practice parameters for the indications for polysomnography andrelated procedures: an update for 2005." Sleep, vol. 28, no. 4, pp. 499-521, Apr 2005 . 